\title{
Face and Emotion Recognition in MCDD Versus PDD-NOS
}

\author{
Catherine M. Herba $\cdot$ Esther de Bruin . \\ Monika Althaus · Fop Verheij · Robert F. Ferdinand
}

Published online: 25 August 2007

(C) Springer Science+Business Media, LLC 2007

\begin{abstract}
Previous studies indicate that Multiple Complex Developmental Disorder (MCDD) children differ from PDD-NOS and autistic children on a symptom level and on psychophysiological functioning. Children with MCDD $(n=21)$ and PDD-NOS $(n=62)$ were compared on two facets of social-cognitive functioning: identification of neutral faces and facial expressions. Few significant group differences emerged. Children with PDD-NOS demonstrated a more attention-demanding strategy of face processing, and processed neutral faces more similarly to complex patterns whereas children with MCDD showed an advantage for face recognition compared to complex patterns. Results further suggested that any disadvantage in face recognition was related more to the autistic features of the PDD-NOS group rather than characteristics specific to MCDD. No significant group differences emerged for identifying facial expressions.
\end{abstract}

Keywords MCDD - Pervasive developmental disorders · PDD-NOS · Face recognition .

Facial expression recognition - Emotion recognition

This work was conducted in the Department of Child and Adolescent Psychiatry, Erasmus Medical Center/Sophia Children's Hospital, Rotterdam.

C. M. Herba $(\bowtie)$ - E. de Bruin · F. Verheij - R. F. Ferdinand Department of Child and Adolescent Psychiatry, Erasmus Medical Center - Sophia Children's Hospital Rotterdam, P.O. Box 2060, Rotterdam 3000 CB, The Netherlands

e-mail: c.herba@erasmusmc.nl

M. Althaus

University Center of Child and Adolescent Psychiatry,

University of Groningen, Groningen, The Netherlands

\section{Introduction}

Social cognition is a crucial component of healthy adjustment, and deficits have been reported in a range of psychiatric disorders in children and adults (for review, see Blair 2003; Phillips et al. 2003a; Walker 1981). A core deficit in children with autism spectrum disorders, or Pervasive Developmental Disorder (PDD) (as defined in the DSM-IV; (APA 1994) relates to social cognition, important aspects of which are, for example, the processing of faces and facial expressions. Encompassed by the overarching category of PDD is the diagnosis of Pervasive Developmental Disorder Not Otherwise Specified (PDD-NOS). Children with PDD-NOS form a heterogeneous group characterized by autistic-like symptoms of varying severity such as marked impairments in social interaction, communication and/or rigid and stereotyped behavior patterns, but fail to meet full criteria for autistic disorder (APA 1994; Walker et al. 2004). Within this heterogeneous group of PDD-NOS children, Cohen and colleagues highlighted the existence of a number of children with disturbances in various areas of functioning such as the regulation of state and arousal (i.e. anxiety and fears), social relations (i.e. detached, aggressive, clingy), and thought disorders (i.e. magical thinking, unusual thoughts, and difficulties in separating fantasy from reality) (Cohen et al. 1986). These children have been described in the past as schizotypal, having borderline disorder, childhood schizophrenia, childhood onset PDD or atypical PDD (for review see Ad-Dab'bagh and Greenfield 2001). The description of this group was refined through a specific set of diagnostic criteria, and the term 'Multiplex Developmental Disorders' (MDD) was proposed (Cohen et al. 1986). Subsequently, the criteria were altered and the term was modified to 'Multiple Complex Developmental Disorder' (MCDD) (Towbin et al. 1993). Although Cohen and colleagues 
positioned MDD within the broader classification of Pervasive Developmental Disorder (PDD), they also recognized the overlap with several other DSM-III (APA 1980) disorders (i.e. Avoidant Disorder, Overanxious Disorder, Schizotypal Disorder) (Cohen et al. 1986). To date, there is little research on these children, however further study is warranted since MCDD children may be at risk for a poor outcome in adulthood including Axis II disorder (Lofgren et al. 1991), and schizophrenia spectrum disorders (van Engeland and van der Gaag 1994). This study aims to directly compare children with MCDD to those with PDDNOS on two measures of social cognition: face recognition and identification of facial expressions. Both of these skills are important to examine given the difficulties in social functioning reported in children with MCDD. Such studies are valuable since any emerging differences in social-cognitive functioning between these two groups of children would further support the validity of MCDD as a separate diagnostic construct. Further validation of the concept of MCDD would emphasize the importance of investigating etiology and efficacy of treatments for MCDD separately from PDD-NOS.

On a symptom level, children with MCDD can be distinguished from other developmental disorders. A study examining the medical charts of children with MCDD, children with autism, and children with externalizing and internalizing disorders, reported that MCDD children, compared to children with autism, were more aggressive, more anxious, showed more psychotic thinking, and suspiciousness. Autistic children were more disturbed in their social interaction and communication and displayed more stereotyped and rigid behavior than MCDD children (van der Gaag et al. 1995). There is also evidence that children with MCDD or autism have elevated levels of formal thought disorder compared to children with Attention Deficit Hyperactivity Disorder (ADHD) and anxiety disorders (van der Gaag et al. 2005). A recent study directly comparing the symptom profiles of children with MCDD and PDD-NOS, reported that the greatest group differences (i.e. largest effect sizes) were found for psychotic thought problems (de Bruin et al. 2007). MCDD children, in comparison to PDD-NOS children, experienced more paranoia, incoherent thoughts and delusions. Furthermore, children with MCDD also had a higher frequency of anxiety disorders and disruptive behavior disorders according to the Diagnostic Interview Schedule for Children (Version IV) (DISC-IV) (i.e. Oppositional Defiant Disorder, and Conduct Disorder) compared to children with PDD-NOS. De Bruin and colleagues (2007) applied research criteria to assess MCDD and PDD-NOS independently of one another, and found that a greater percentage of children with PDD-NOS met criteria for a diagnosis of autism or autism spectrum disorder (ASD) and scored higher on measures of reciprocal social interaction and communication deficits as assessed using the Autism Diagnostic Observation Schedule-Generic (ADOS-G) (Lord et al. 1999). Findings suggest that children with MCDD, compared directly to those with PDD-NOS, can be differentiated on various dimensions, one of which (i.e. psychotic thought problems) might resemble difficulties experienced by adults with schizophrenia.

Children with MCDD also demonstrate psychophysiological differences compared to children with autism, ADHD, dyslexia, and normally developing children as assessed by Event-Related Potentials (ERP) obtained during performance on a visual oddball task (Kemner et al. 1999). There is evidence for a blunted cortisol response to psychosocial stress compared to normally developing children (Jansen et al. 2000a) and children with autism (Jansen et al. 2003). Although both MCDD and autistic children are characterized by abnormal reactions to their social environment, group differences in cortisol response to psychosocial stress suggests that the disorders may have different etiological backgrounds or perhaps may be different neurobiological conditions. A blunted response to psychosocial stress among children with MCDD could also be partly due to higher levels of comorbid conduct disorders. Similar blunted cortisol responses to psychosocial stress have also been reported in adults with schizophrenia (Jansen et al. 1998, 2000b, 2003). This suggests that children with MCDD may possess a biological vulnerability to this disorder that may not be evident among children with PDD's (at least autism).

Findings to date on the symptom and biological/psychophysiological profiles suggest that children with MCDD form a group who may not be well placed under the umbrella of the PDD's, since there is evidence that they may be at risk for developing schizophrenic spectrum disorders later in life. Despite the differences seen among children with MCDD compared to other clinical groups on a symptom, psychophysiological, and neurobiological level, no studies to our knowledge have assessed socialcognitive functioning in these children. The only study to investigate a related area, examined formal thought disorder in MCDD children compared with other clinical groups (van der Gaag et al. 2005). Although children with MCDD may demonstrate similar core social deficits as seen in children with PDD-NOS, parallels between the symptoms and biological reactivity in children with MCDD, and adults with schizophrenia spectrum disorders, suggest that a different pattern of performance on social cognitive tasks might emerge compared to children with PDD-NOS. We aimed to examine whether children with MCDD could be differentiated from children with PDD-NOS on two important aspects of social cognition: face recognition and the identification of facial expressions. 
Face Recognition

Serra and colleagues provided empirical evidence that a more time-consuming, controlled, attention-demanding strategy may characterize face processing in children with PDD-NOS by demonstrating that children with PDD-NOS were significantly slower in face recognition than agematched normally developing children while the recognition of abstract visuo-spatial patterns did not discriminate between the groups (Serra et al. 2003). Klin and colleagues studied face recognition in children with autism, PDDNOS, and non-PDD disorders (Klin et al. 1999). Findings revealed a specific deficit in face recognition in children with autism (which could not be attributed to general cognitive ability), but no specific face recognition deficit in children with PDD-NOS. However, these authors did not investigate processing times.

To our knowledge, no studies have examined face processing in children with MCDD, nor have any directly compared children with MCDD to those with PDD-NOS. We can only speculate on the results for MCDD children. However, if children with MCDD are distinct from those with PDD-NOS, we might expect them to show a different pattern of face recognition compared to children with PDD-NOS only (Serra et al. 2003), which might be characterized by less controlled time-consuming processing.

\section{Identification of Facial Expression}

Facial expression recognition has not been previously examined in children specifically diagnosed with MCDD. Considering that children with MCDD have been previously described as schizotypal or having a diagnosis of childhood schizophrenia (see Ad-Dab'bagh and Greenfield 2001), a study that most closely approximates the investigation of facial expression recognition in MCDD is one examining emotion expression recognition in children with schizophrenia, aggression, anxiety/depression, and normally developing children (Walker 1981). Walker (1981) reported that children with schizophrenia were less accurate in recognizing emotional expressions compared to the other groups.

Deficits in identifying facial expressions have been reported in children with PDD (mainly autism) (Castelli 2005; Celani et al. 1999; Hobson et al. 1988). Although a review by Blair (2003) on facial expression recognition in neuro-cognitive disorders noted that once participants with autism were matched to controls on mental age, impairments in emotion expression recognition disappeared. Few studies have probed emotion processing in children with PDD-NOS. One study comparing normally intelligent PDD-NOS children with school children on emotion recognition (i.e. face, posture, and gesture recognition), found no differences between the two groups (Serra et al. 1998).

Based on the above findings, and given the fears and anxieties inherent to the classification of MCDD, one might predict specific differences, particularly in the identification of threatening expressions (i.e. fearful and angry expressions) compared to children with PDD-NOS. This is based on previous research indicating that adults and children with elevated levels of anxiety demonstrate biases in processing anger/threatening expressions (Hadwin et al. 2003; Mogg et al. 2004).

\section{Aims and Predictions of the Study}

If robust social cognitive differences would emerge, in light of the symptom profile and biological/psychophysiological differences between the groups, there would be further evidence for recognizing a subcategory of MCDD within the DSM-V as already suggested by others (van der Gaag et al. 1995). Considering MCDD as qualitatively distinct from PDD-NOS has implications for treatment. Viewing MCDD as a subgroup of PDD-NOS may focus treatment on the improvement of social skills, whereas if the emphasis in MCDD was on the thought disorder and its relation to psychotic development as well as on anxiety symptoms, a more medication-focused approach might be preferred. However, if social cognitive skills were quite the same, such a finding could also suggest that the diagnostic boundaries of the DSM-categories are weak: people with schizoid/psychotic symptoms might have a neurobiological condition different from that of people with only PDDsymptoms though sharing important PDD core features. Such a finding would stress the necessity for profiling patients along various dimensions including their psychophysiological responsiveness and (social) cognitive capacities.

We aimed to address whether children with MCDD significantly differ from children with PDD-NOS on two facets of social-cognitive skills: face recognition and the identification of facial expressions. Children with MCDD and PDD-NOS were carefully selected using explicitly outlined research criteria. Based on previous research:

(1) We predicted that children with MCDD would differ from those with PDD-NOS on recognizing neutral faces in comparison to complex patterns. More specifically, if indeed children with MCDD are not well-placed under the PDD's, we would expect them to be faster and more accurate on face recognition compared to children with PDD-NOS, but perform similarly on especially complex pattern recognition. 
(2) Children with MCDD would show differences in the identification of facial expressions, particularly a bias toward processing fear and anger expressions, as compared to children with PDD-NOS. Any significant differences found should not be explained by differences in mental age.

\section{Methods \\ Participants}

The study sample was selected from 503 children, aged 612 years old, who were consecutively referred to the outpatient department of child and adolescent psychiatry, between July 2002 and September 2004. Referrals were comprised of a large variety of child psychiatric disorders (externalizing disorders, internalizing disorders, PDDs). Research criteria for MCDD and PDD-NOS were rated incompletely for $12(2.4 \%)$ children, who were excluded from further analyses. Complete MCDD and PDD-NOS criteria were rated for 491 children.

Twenty-nine $(5.9 \%)$ children met research criteria for a diagnosis of MCDD. Eleven children (44\%) meeting MCDD research criteria also met research criteria for PDD-NOS. These children were placed in the MCDD group. The parents of four of these children refused to participate in the study. Seventy-nine children met research criteria for a diagnosis of PDD-NOS without meeting research criteria for MCDD (PDD-NOS group). These children did not meet DSM-IV criteria for autism or Asperger syndrome. Children with an IQ score of less than 70 were not administered the neuropsychological battery; two children in the MCDD group, and 13 children in the PDD-NOS group were not administered the neuropsychological tasks on this basis. A further two children meeting MCDD criteria had missing data for the face recognition (FR) task and identification of facial expressions task (IFE). Thus, the MCDD group included a total of 21 children. In addition to the children without neuropsychological data due to a low IQ, five children in the PDD-NOS group were missing data for the FR task, and four were missing data for the IFE task, resulting in a total of 61 PDD-NOS children with FR task data, and 62 children with IFE task data.

\section{Ethics}

Participation was voluntary, and informed consent was signed by all parents/caretakers prior to participation in the study. Children who were 12 years old also signed the consent forms themselves. The Medical Ethics Committee of the Erasmus Medical Center approved the study.

\section{MCDD and PDD-NOS Research Criteria}

Explicit research criteria for MCDD and PDD-NOS were used (see Table 1) (Buitelaar et al. 1999a). Nine different child and adolescent psychiatrists were involved in rating these research criteria. Ratings were based on semi-structured interviews with the parents/caretakers and individual psychiatric observation of the child. Assessment information spanned from early development to current level of social, communicative, and adaptive functioning. School, relevant medical, and psychological assessment information were obtained as well. Immediately after all diagnostic procedures had been completed, MCDD and PDD-NOS research criteria were ticked as present or absent, and subsequently an algorithm, of which the rater was unaware, was used to determine whether the thresholds for research diagnoses of MCDD and PDD-NOS were met. For a detailed review of the development of the MCDD and PDD-NOS research criteria see Buitelaar and van der Gaag (1998).

An interrater reliability study was conducted for 30 randomly selected children (27\%). Two clinicians independently rated all MCDD and PDD-NOS research criteria. Agreement between the raters on the presence or absence of a PDD-NOS diagnosis was good $(\kappa=.62)$. Agreement for MCDD diagnosis could not be calculated, as MCDD did not occur once in this subsample.

\section{Materials}

\section{Procedure}

Children were assessed on two occasions, separated by a week. Testing was conducted in a quiet room in the outpatient department of the hospital. On the first occasion, the full Weschsler Intelligence Scale for Children (WISC-R) was administered, taking on average, $2 \mathrm{~h}$ per child. The social-cognitive tasks were administered on the second visit the following week as part of a larger neuropsychological battery taking approximately one and a half hours. Children were always tested in the morning to minimize the effects of fatigue and to maximize concentration.

\section{Intelligence: Weschsler Intelligence Scale for Children} (WISC-R)

The full WISC-R (revised for use in the Netherlands) was administered to the children. Based on the full-scale IQ 
Table 1 Research criteria used to identify children with MCDD and PDD-NOS (from Buitelaar and van der Gaag 1998)

$\mathrm{MCDD}^{1} \quad \mathrm{PDD}^{1} \mathrm{NOS}^{2}$

(1) Impaired regulation of affective states and anxieties

(a) Unusual or peculiar fears and phobias, or frequent idiosyncratic or bizarre anxiety reactions

(b) Recurrent panic episodes, or flooding with anxiety

(c) Episodes of behavioral disorganization punctuated by markedly immature, primitive, or violent behaviors

(2) Impaired social behavior

(a) Social disinterest, detachment, avoidance, or withdrawal

(b) Markedly disturbed and/or ambivalent attachments

(3) The presence of thought disorder

(a) Irrationality, magical thinking, sudden intrusions on normal thought process, bizarre ideas, neologism, repetition of nonsense words

(b) Perplexity and easy confusability. overvalued ideas, including fantasies of omni-potence, paranoid preoccupations, overengagement with fantasy figures, referential ideation
$A^{2}$. (1) Qualitative impairment in social interaction

(a) Marked impairment in the use of multiple nonverbal behaviors, such as eye-to-eye gaze, facial expression, body postures, and gestures to regulate social interaction

(b) Failure to develop peer relationships appropriate to developmental level

(c) A lack of spontaneous seeking to share enjoyment, interests, or achievements with other people (e.g. by a lack of showing, bringing, or pointing out objects of interest)

(d) Lack of social and emotional reciprocity

(2) Qualitative impairments in communication

(a) In individuals with adequate speech, marked impairment in the ability to initiate or sustain a conversation with others

(b) Stereotyped and repetitive use of language or idiosyncratic language

(3) Restricted repetitive and stereotyped patterns of behavior, interests, and activities

(a) Stereotyped and repetitive motor mannerisms (e.g. hand or finger flapping or twisting, or complex whole-body movements)

B. Does not meet criteria for autistic disorder or for other specific pervasive developmental disorder

${ }^{1}$ A total of 5 or more items from 1,2, and 3, with at least one item from (1), one item from (2), and one item from (3)

2 A total of 3 or more items from (1), (2), and (3), with at least one item from (1)

score, each child's mental age was computed using the following formula: Mental age $=($ age $*$ full scale IQ score)/100. Mental age (MA) was included as a covariate since it has been shown to be an important mediator for group differences in social cognition (see Buitelaar et al. 1999b; Happe 1995).

\section{Baseline Speed (BS)}

A simple reaction time task was employed to obtain a baseline measure for the speed (BS) of responding with the response key to ensure children understood how to respond using the response key. Children were required to press a key with the index finger of their dominant hand when a square was presented. Thirty-two trials were administered. This task was a subtest of the computerized Amsterdam Neuropsychological Tasks (ANT version 2.1; de Sonneville 1999). The total baseline speed and the standard deviation (SD) of the BS were calculated. The SD provides a measure of the variability of performance. A higher SD could indicate less attention to the task.

\section{Face Recognition}

The Face Recognition (FR) subtest of the ANT 2.1 battery (de Sonneville 1999) was selected to measure the speed and accuracy of recognizing neutral faces. This task has previously been administered in studies assessing face recognition in children with PDD-NOS (Serra et al. 2003) and in normally developing children (de Sonneville et al. 2002). In the FR task, a target (neutral) face was presented for $2.5 \mathrm{~s}$. Following the presentation of the target face, a set of four photographs of individuals was presented and children were required to indicate (using a two-key response panel) whether or not the target individual appeared in the set of four (see Fig. 1a). The sex and age category of the target (i.e. boys, girls, men or women) matched those of the subsequently shown set of four faces to be judged. In half of the trials (i.e. 20), the target individual did appear in the set of four and participants were required to press a 'yes' key ('target' condition), and in 20 trials the target individual did not appear in the subsequent set of four, requiring participants to press the 'no' key ('non-target' condition). Reaction time (RT) data and accuracy (assessed by calculating the proportion of correct trials out of the maximum score) were calculated for target and non-target conditions.

\section{Pattern Recognition}

A subtest of the ANT 2.1 battery assessing pattern recognition (PR) was administered. The task consisted of two 
conditions, one containing visuo-spatial patterns that are dissimilar and hence easily distinguishable (easy condition), and the other containing complex patterns that are quite similar and therefore hardly distinguishable (complex condition) (see Fig. 1b). The same manner of responding was used as in the FR task, and the same four outcome variables were generated: RT and accuracy for target and non-target conditions. As with the FR task, there were 20 trials for the target condition and 20 trials for the non-target condition, each for the easy condition and the complex condition. Easy and complex patterns as target and nontarget trials were presented in a random manner.

\section{Identification of Facial Expressions}

The "Identification of Facial Expressions"(IFE) subtest of the ANT 2.1 was employed to probe emotion processing (see Fig. 2). Children were required to respond as to whether a face displayed a particular target emotion (by pressing a "yes" button) or not (by pressing the "no" button). Four conditions were administered, each corresponding to a target emotion (i.e. happy, sad, anger, and fear). For each condition, children were instructed to focus on a particular emotion, and to respond whether the face demonstrated that particular emotion or not (i.e. for the happy condition, children were to respond "yes" if the face was happy or to press the "no" button if the face displayed a different emotion). Each emotion condition consisted of 40 trials, 20 of which were the target emotion (requiring a "yes" response) and 20 of which were a random selection of other emotions (requiring a "no" response). The images were digitized photographs of four adult identities (two men and two women). Four outcome variables were calculated for each emotion category: (1) RT for target condition (when the target emotion is presented) (2) RT for non-target condition (when the target emotion is not presented) (3) Accuracy in the target condition (4) Accuracy in the nontarget condition. Accuracy (calculated for target and nontarget conditions separately) was assessed by calculating the proportion of correct trials out of the maximum score.

\section{Statistical Analysis}

A series of multivariate General Linear Models (GLM) was conducted to compare children with MCDD to those with PDD-NOS-only on the various outcome variables of BS, PR, FR, and IFE tasks. For each task (except for the BS task which yielded reaction times only), separate repeatedmeasures analyses of covariance (ANCOVAs) were performed on the measures for accuracy and speed of

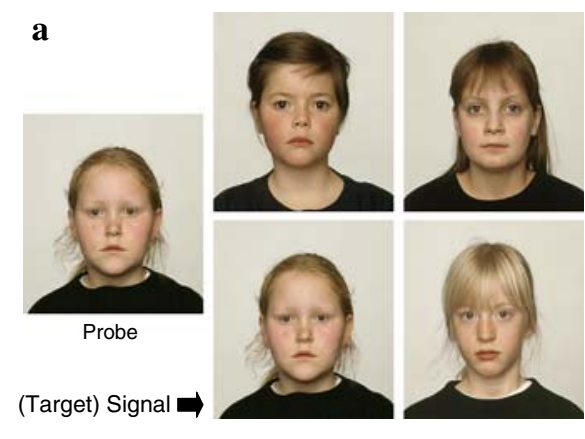

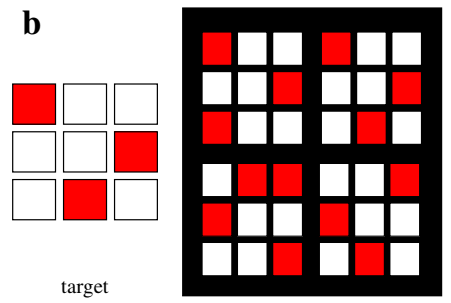

(1) Complex PR

Target condition

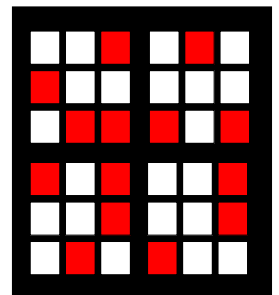

(2) Complex PR

Non-target condition

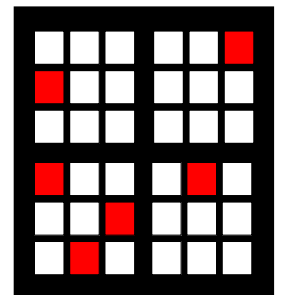

(3) Easy PR

Target condition

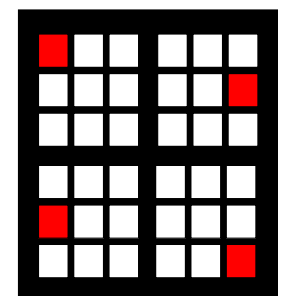

(4) Easy PR

Non-target condition
Fig. 1 (a) Face Recognition (FR) task. A neutral face (target stimulus) is presented for $2,500 \mathrm{~ms}$, followed by a $500 \mathrm{~ms}$ delay. A display set of four neutral faces is then presented. The child must indicate (using a two-button response key) whether the target face is present in the display set (target condition) or not (nontarget condition). Reaction time and accuracy (proportion correct, with a maximum value of 1) data were calculated for target and non-target conditions. (b) Pattern Recognition (PR) task. The children must indicate whether a target pattern is present in one of four patterns presented in a display set. Presentation time parameters and outcome variables are the same as in the FR task. Two PR tasks are presented: (1) patterns involving a similar level of complexity to the FR task (i.e. Complex PR), (2) patterns of a dissimilar (i.e. Easy PR) level of difficulty compared to the FR task 

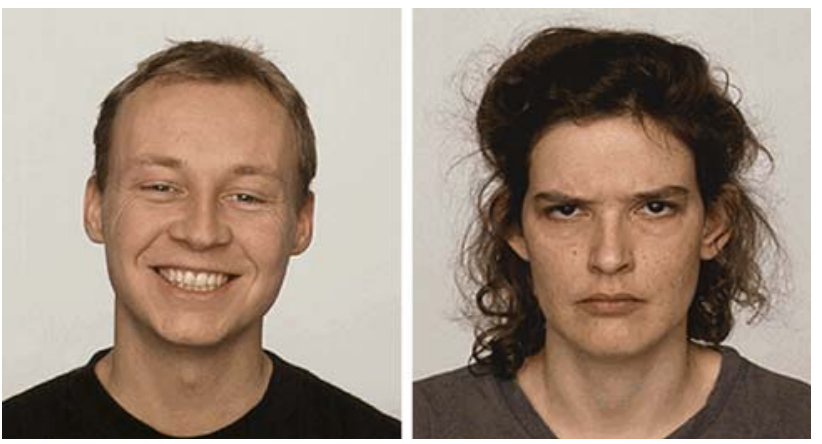

Fig. 2 Examples of different expressions in the Identification of Facial Expressions (IFE) task. Children are presented with four different tasks (each corresponding to one of four target emotions: happy, sad, anger, and fear). For each task, children are required to focus on a particular emotion, and to judge whether the face displays a specific target emotion. The target consists of an adult face expressing one of four emotions. When the face matches the emotion a 'yes' response is required, when the face does not match the emotion, a 'no' response is required. A total of 40 trials per emotion condition were presented, with half of those trials requiring a 'yes' response (target), and half requiring a 'no' response (nontarget). RT and accuracy (i.e. proportion correct) for target and non-target conditions were calculated

processing. Assumptions underlying the use of parametric statistics were examined for each outcome variable. Where assumptions of normality were violated, transformations were applied. Only variables representing accuracy (for target and non-target conditions) were transformed. For these, we applied the arc sin transformation, since this is appropriate for proportional data (Howell 1997). Following these transformations, data were appropriate for the use of parametric statistics. For the repeated-measures ANCOVA's, Wilk's lambda and corresponding $F$-statistics and significance are presented where Mauchly's test of sphericity was not significant. Where this test was significant, the corrected Greenhouse-Geisser degrees of freedom and significance levels are presented. Two-tailed tests were used. Effect sizes (small: $\geq 0.02$ and $\leq .06$; medium: $>.06$ and $\leq 0.13$; large: $\geq 0.14$ ) were estimated using partial Eta squared $\left(\eta_{\mathrm{p}}^{2}\right)$ (Stevens 1992).
If any significant group differences were found, we repeated the analyses while covarying for the effects of mental age (MA) to exclude the possibility that the differences could be explained by variations in general intelligence.

\section{Results}

\section{Group Characteristics}

Children in the MCDD group did not significantly differ from the PDD-NOS group on age $(F(1,78)=2.29$, $p=0.14$ ), sex distribution (Fisher's Exact $=1.00$ ), VIQ $(F(1,82)=0.22, \quad p=0.64), \quad$ PIQ $\quad(F(1,82)=0.59$, $p=0.44)$, FIQ $(F(1,82)=0.04, p=0.84)$ or MA $(F(1$, $78)=1.07, p=0.30)$. Means $(\mathrm{SD})$ are presented in Table 2. There were no significant group differences in baseline speed $(\mathrm{BS})(F(1,79)=0.21, p=0.65)$ or $\mathrm{SD}$ of BS $(F(1,79)=0.59, p=0.44)$. This indicates that children in the PDD-NOS group were not significantly different from those in the MCDD group in terms of their basic ability to use the response key or basic attention to the task.

\section{Face Recognition (FR) Compared with Pattern} Recognition (PR)

Our first hypothesis was that children with PDD-NOS would be less accurate and slower in processing neutral faces compared to children with MCDD. Such a group difference was not expected for the recognition of abstract visuo-spatial patterns. Means and standard deviations (SD) for accuracy and speed of performance on both the face recognition (FR) and pattern recognition (PR) task are presented in parts 1 and 2 of Table 3, and generally indicate a slower speed of processing in the PDD-NOS group (for both face recognition and pattern recognition) while differences in accuracy appear less pronounced.
Table 2 Frequencies, Means (SD) for the MCDD and PDDNOS groups for age, sex, and IQ

\begin{tabular}{llll}
\hline & $\begin{array}{l}\text { PDD-NOS, } \\
N=62 \\
\text { Mean (SD) }\end{array}$ & $\begin{array}{l}\text { MCDD (all with } \\
N=21\end{array}$ & $\begin{array}{l}\text { MCDD), 'Pure' MCDD subsample, } \\
N=13\end{array}$ \\
\hline $\begin{array}{l}\text { Chronological age } \\
\quad \text { years) }\end{array}$ & $9.22(1.82)$ & $9.89(1.49)$ & $9.87(1.47)$ \\
$\begin{array}{l}\text { Male/Female }(n) \\
\text { Mental age (years) }\end{array}$ & $54 / 8$ & $18 / 3$ & $10 / 3$ \\
$\begin{array}{l}\text { IQ: } \text { WISC- } R \\
\text { Verbal IQ }\end{array}$ & $9.83(2.10)$ & $9.35(1.60)$ & $9.43(1.77)$ \\
Performance IQ & $95.54(14.26)$ & $97.33(17.75)$ & $98.77(21.31)$ \\
Total IQ & $97.57(16.64)$ & $94.52(12.55)$ & $94.46(10.15)$ \\
\hline
\end{tabular}


To test our hypothesis, two types of repeated measures analyses were conducted, each on our measures of accuracy and speed of processing separately. In the first type we compared the easy condition of the PR task with the performance of the FR task, in the second type we compared the complex condition of the PR with the FR task. Each analysis included two within-subjects variables: (1) "task" (FR versus PR) and (2) "response type" (target versus nontarget). The between-subjects variable was group. Significant group by task interactions would indicate that the groups differ in their manner of processing neutral faces as compared to abstract patterns.

Concerning performance on the easy condition of pattern recognition as compared to face recognition, there were no significant interactions involving group (i.e. group*task*response type or group*task) nor a significant main effects of group for either accuracy or speed of processing. Irrespective of group, a significant interaction between task and response type for RT (Wilk's lambda = $\left.0.26, F(1,80)=231.10, p<0.001 ; \eta_{\mathrm{p}}^{2}=0.74\right)$, indicated that children took substantially longer to perform the FR task compared to easy PR, and this effect was particularly strong for the non-target condition.

Comparison of the complex condition of the PR task with face recognition revealed a significant interaction between group, task (PR versus FR), and response type (target versus non-target) (Wilk's lambda $=0.94, \quad F(1,80)=5.07$, $p=0.03 ; \eta_{\mathrm{p}}^{2}=0.06$ ). Children with PDD-NOS were less accurate in recognizing the presence of a target face in the response set compared to children with MCDD, whereas this difference was not evident when children had to recognize a target pattern amongst hardly distinguishable other
Table 3 Means (SD) for face recognition, pattern recognition, and identification of facial expressions tasks

\begin{tabular}{|c|c|c|c|}
\hline Task variables & $\begin{array}{l}\text { PDD-NOS } \\
\text { RT (ms): Mean (SD }\end{array}$ & $\begin{array}{l}\text { MCDD-all } \\
\text { Proportion accurat }\end{array}$ & $\begin{array}{l}\text { Pure MCDD } \\
\text { Mean (SD) }\end{array}$ \\
\hline Face recognition (FR) & $N=62$ & $N=21$ & $N=13$ \\
\hline Targets & $\begin{array}{l}2155.29(625.54) \\
0.76(0.17)\end{array}$ & $\begin{array}{l}1862.71(469.08) \\
0.83(0.17)\end{array}$ & $\begin{array}{l}1853.12(468.23) \\
0.86(0.11)\end{array}$ \\
\hline Non-targets & $\begin{array}{l}2751.15(730.77) \\
0.84(0.12)\end{array}$ & $\begin{array}{l}2599.61(697.01) \\
0.84(0.14)\end{array}$ & $\begin{array}{l}2701.30(742.33) \\
0.82(0.15)\end{array}$ \\
\hline Pattern recognition (PR) & $N=61$ & $N=21$ & $N=13$ \\
\hline Easy PR: Targets & $\begin{array}{l}1826.3(519.12) \\
0.96(0.07)\end{array}$ & $\begin{array}{l}1689.90(509.77) \\
0.97(0.05)\end{array}$ & $\begin{array}{l}1785.92(544.13) \\
0.98(0.02)\end{array}$ \\
\hline Easy PR: Non-targets & $\begin{array}{l}1449.47(470.34) \\
0.85(0.23)\end{array}$ & $\begin{array}{l}1332.83(356.20) \\
0.89(0.17)\end{array}$ & $\begin{array}{l}1364.01(392.80) \\
0.87(0.20)\end{array}$ \\
\hline Complex PR: Targets & $\begin{array}{l}2627.03(723.78) \\
0.83(0.23)\end{array}$ & $\begin{array}{l}2536.89(763.48) \\
0.80(0.26)\end{array}$ & $\begin{array}{l}2656.76(886.98) \\
0.78(0.29)\end{array}$ \\
\hline Complex PR: Non-targets & $\begin{array}{l}3318.54(984.58) \\
0.76(0.20)\end{array}$ & $\begin{array}{l}3084.97(845.74) \\
0.84(0.13)\end{array}$ & $\begin{array}{l}3129.71(1016.1) \\
0.83(0.14)\end{array}$ \\
\hline Identification of Facial Expressions (IFE) & $N=62$ & $N=21$ & $N=13$ \\
\hline Happy: Targets & $\begin{array}{l}1001.03(358.12) \\
0.92(0.09)\end{array}$ & $\begin{array}{l}977.91(347.91) \\
0.95(0.04)\end{array}$ & $\begin{array}{l}1103.95(380.76) \\
0.95(0.05)\end{array}$ \\
\hline Happy: Non-targets & $\begin{array}{l}1322.07(482.98) \\
0.95(0.06)\end{array}$ & $\begin{array}{l}1145.38(352.43) \\
0.94(0.06)\end{array}$ & $\begin{array}{l}1231.97(394.17) \\
0.95(0.06)\end{array}$ \\
\hline Sad: Targets & $\begin{array}{l}1345.73(419.14) \\
0.69(0.25)\end{array}$ & $\begin{array}{l}1217.74(414.07) \\
0.77(0.21)\end{array}$ & $\begin{array}{l}1311.15(467.99) \\
0.81(0.15)\end{array}$ \\
\hline Sad: Non-targets & $\begin{array}{l}1691.06(657.64) \\
0.78(0.19)\end{array}$ & $\begin{array}{l}1482.22(412.78) \\
0.86(0.13)\end{array}$ & $\begin{array}{l}1569.99(382.69) \\
0.86(0.14)\end{array}$ \\
\hline Anger: Targets & $\begin{array}{l}1221.14(513.73) \\
0.73(0.20)\end{array}$ & $\begin{array}{l}1115.36(275.29) \\
0.75(0.21)\end{array}$ & $\begin{array}{l}1172.91(296.49) \\
0.79(0.19)\end{array}$ \\
\hline Anger: Non-targets & $\begin{array}{l}1538.94(614.53) \\
0.89(0.15)\end{array}$ & $\begin{array}{l}1417.10(361.06) \\
0.89(0.13)\end{array}$ & $\begin{array}{l}1535.36(347.95) \\
0.87(0.15)\end{array}$ \\
\hline Fear: Targets & $\begin{array}{l}1351.81(573.98) \\
0.79(0.18)\end{array}$ & $\begin{array}{l}1301.85(491.01) \\
0.78(0.24)\end{array}$ & $\begin{array}{l}1327.11(586.66) \\
0.80(0.26)\end{array}$ \\
\hline Fear: Non-targets & $\begin{array}{l}1476.66(519.16) \\
0.82(0.20)\end{array}$ & $\begin{array}{l}1321.81(300.87) \\
0.87(0.14)\end{array}$ & $\begin{array}{l}1377.08(355.14) \\
0.87(0.15)\end{array}$ \\
\hline
\end{tabular}


patterns (see Fig. 3). This interaction bordered on significance after controlling for MA $\left(p=0.05 ; \eta_{\mathrm{p}}^{2}=0.05\right)$. Such results did not emerge for speed of processing: there were no significant interactions; group*task*target $(p=0.91$; $\left.\eta_{\mathrm{p}}^{2}<0.01\right)$ or group*task $\left(p=0.73 ; \eta_{\mathrm{p}}^{2}<0.01\right)$, nor was there a significant main effect of group $\left(p=0.23 ; \eta_{\mathrm{p}}^{2}=\right.$ 0.02). Irrespective of group, children processed faces faster than complex patterns (Wilk's lambda $=0.64, \quad F(1$, 80) $\left.=44.85, p<0.001 ; \eta_{\mathrm{p}}^{2}=0.36\right)$.

Our first hypothesis was therefore partially supported: children with PDD-NOS were less accurate than children with MCDD in recognizing that a neutral face was present in the response set, but this effect was not seen when they were asked to note whether an abstract pattern was present amongst similar ones (i.e. complex condition). There was, however, no group difference in task dependent RTs.

\section{Identification of Facial Expressions (IFE) Task}

Our second hypothesis was that children with MCDD would demonstrate differences in the identification of

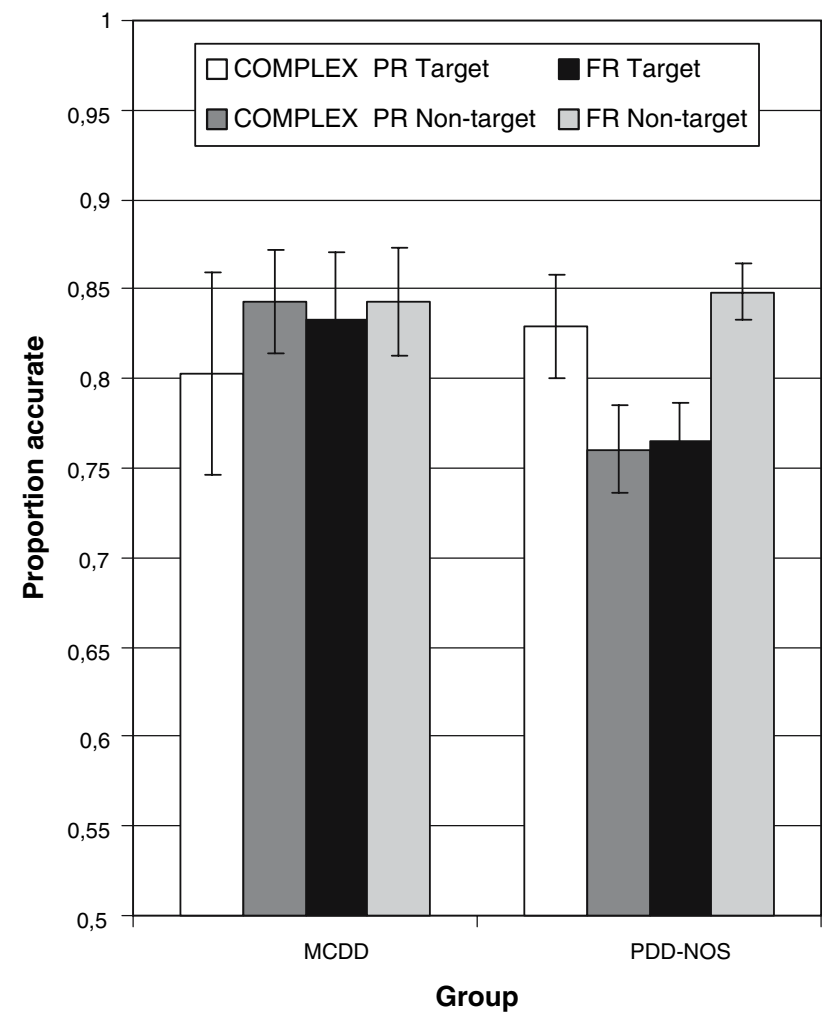

Fig. 3 Accuracy of PDD-NOS versus MCDD children for face recognition (FR) and complex pattern recognition (PR). This figure is based on raw (untransformed) error rates, without covarying for MA. Error bars represent standard error of the mean facial expressions compared to those with PDD-NOS, particularly in the identification of fear and anger expressions. Means and standard deviations (SD) for both measures of speed and accuracy are presented in the third part of Table 3 showing a generally slower speed of processing in the PDD-NOS group while differences in accuracy appear less pronounced. To examine group differences in children's identification of facial expressions, two repeated-measures analyses were conducted (one for accuracy and the other for speed of processing). The within-subjects factors included (1) emotion-category (four levels: happy, sad, anger, fear) and (2) response type (target versus non-target). The between-subjects factor was group.

Contrary to our expectations, children with MCDD did not significantly differ from those with PDD-NOS on emotion-processing; there were no significant main effects of group for accuracy $(p=0.22)$ or for RT $(p=0.18)$, nor any significant interactions between group*emotion-category for either accuracy $(p=0.16)$ or RT $(p=0.98)$ data. We therefore found no support for our second hypothesis that children with MCDD would process facial expressions of emotion (particularly anger and fear faces) differently compared to children with PDD-NOS.

\section{Reanalyses Including 'Pure' MCDD Versus PDD-NOS Children}

Because approximately $40 \%$ of the children with MCDD also fulfilled research criteria for PDD-NOS, it is plausible that MCDD status might have been confounded with PDDNOS. To ensure that the results were not simply due to the overlap between children with MCDD, and PDD-NOS, we repeated the above analyses, however this time comparing a sub-sample of the MCDD children who fulfilled research criteria for MCDD but not PDD-NOS ('pure MCDD'; $n=13)$, to children with PDD-NOS $(n=61)$. For emotionprocessing (i.e. Identification of Facial Expression task), similar results were obtained as with the larger MCDD group; no significant results involving group ('pure MCDD' versus PDD-NOS) emerged. Yet, re-analysis of the face versus pattern recognition performances revealed a group difference not previously found. This difference relates to the comparison between complex pattern recognition and face recognition; the significant interaction between task*response type*group previously found for only the accuracy data now emerged for RT data as well (Wilk's lambda $=0.94, F(1,72)=4.77, p=0.03 ; \eta_{\mathrm{p}}^{2}=$ 0.06). Children with PDD-NOS compared to the 'pure' MCDD group were slower in recognizing faces in the target condition, whereas no such group difference was found for complex pattern recognition. The interaction remained significant after adjusting for the effect of MA 
$\left(p=0.04 ; \eta_{\mathrm{p}}^{2}=0.06\right)$. All other results were similar when reanalyzed using this more 'pure' MCDD group.

\section{Discussion}

Previous research suggests that children with MCDD may form a group of children that is, based on symptom, biological, and psychophysiological profiles, welldistinguishable from those with autism, externalizing, and internalizing disorders. This group, though possibly at risk for poor prognosis in adulthood, including schizophrenia spectrum disorders, is currently subsumed under the larger, heterogeneous category of PDD-NOS. Yet, there is debate as to whether this group should be considered a separate diagnostic category within DSM-V (van der Gaag et al. 1995). To our knowledge, only one other study has directly compared children with PDD-NOS to those with MCDD, examining symptom differences between the two groups of children (de Bruin et al. 2007). The present study contributes to literature on this under-studied and somewhat controversial diagnosis, by examining whether children with MCDD can be differentiated from children with PDDNOS on two domains of social-cognitive functioning; face recognition and the identification of facial expressions. The ability to recognize individuals, and to process the emotional cues of others quickly and accurately is a crucial component of social functioning and development (for reviews, see Blair 2003; Herba and Phillips 2004).

\section{Face Recognition}

Our first hypothesis postulated that children with PDDNOS would demonstrate poorer performance in face recognition than children with MCDD, but that the two groups would perform similarly on detecting complex patterns. To test this hypothesis we adopted an approach used by Serra and colleagues (2003) which provided evidence that children with PDD-NOS may use a more attention-demanding strategy of face processing compared to typically developing children who were suggested to process faces more automatically. In that study, children with PDD-NOS showed an only small discrepancy in speed of processing complex patterns and faces, whereas typically developing children showed a greater advantage for face recognition compared to pattern recognition. We therefore predicted that children with MCDD, if indeed distinct from those with PDD-NOS, would demonstrate a more specific advantage for face recognition compared to complex pattern recognition, whereas children with PDD-NOS would show a smaller difference between processing faces and complex patterns. Our results are somewhat consistent with this hypothesis. When comparing easy pattern recognition with the recognition of unfamiliar faces, no group differences emerged; overall, children were faster and more accurate to recognize easy patterns compared to faces. However, when comparing the recognition of complex patterns with the recognition of faces, children with PDDNOS appeared to process faces more similarly to complex patterns especially in the target condition whereas children with MCDD demonstrated greater accuracy for processing faces compared to complex patterns. This effect remained significant after adjusting for MA. Moreover, when investigating the 'pure MCDD' group (children who met criteria for MCDD, but not PDD-NOS), the advantage for face recognition could be shown to also exist for their speed of processing. These results suggest that MCDDspecific characteristics on their own (see Table 1: impaired regulation of affective states, impaired social behavior, and thought disorder) might not be associated with a more attention-demanding strategy of face recognition. In contrast, children with PDD-NOS, consistent with their symptoms of impaired non-verbal behavior, lack of social and emotional reciprocity, and lack of spontaneous affiliation with other people, appeared to process facial information in a way quite similar to how they process complex patterns. De Bruin and colleagues (2007), using data from the same participants as in the present study, reported that children in the MCDD group (36.0\%) fulfilled criteria for ADOS-G classifications of autism or autism spectrum to a lesser extent compared to children in the PDD-NOS group (62.2\%). Hence, the more time-consuming and therefore attention-demanding strategy of face processing described by Serra and colleagues and seen in our group of children with PDD-NOS could be due to the more 'autistic' characteristics of our PDD-NOS group. Face recognition appeared to be less attention-demanding in our MCDD group that did not meet the diagnostic criteria for a PDD-NOS. Our results therefore suggest that MCDD symptoms are associated with disadvantages in especially the speed of recognizing unfamiliar faces only to the extent to which they are accompanied by social problems severe enough to meet the diagnostic criteria for PDD-NOS.

\section{Identification of Facial Expressions}

Given the extensive fears and anxieties inherent to the diagnosis of MCDD compared to autism or PDD-NOS, we expected significant group differences to be evident in emotion-processing. We predicted that these group differences would be particularly marked for identifying facial expressions of fear and anger expressions, since previous research has indicated that aberrant patterns in processing 
certain emotions are specific to the symptoms of the particular disorder (Phillips et al. 2003a), and that adults and children with high levels of anxiety demonstrate biases toward processing threatening facial expressions (Hadwin et al. 2003; Mogg et al. 2004). Yet, we found no support that children with MCDD differed significantly from those with PDD-NOS in identifying fear or anger expressions, nor for any of the other emotion-categories we investigated. Taking the model of emotion-processing by Phillips and colleagues proposing the following three main components (Phillips et al. 2003b): (1) identification of the emotional significance of a stimulus; (2) production of an affective state; and (3) regulation of an affective state, we must note that only the first component was tested in the current paper. Evidently, the notable differences between children with MCDD and those with PDD-NOS found on a symptom level (de Bruin et al. 2007) do not translate to a more basic level of emotion recognition. Differences between the two groups in the production and regulation of affective states are nevertheless quite likely, since especially children with MCDD appear to have substantial difficulties regulating their affective state. Future work should incorporate tasks that allow for the examination of each of the three of the above-mentioned components of emotion-processing. In order to provoke changes in affective state such a task should take into account real-life situations or experiences that may trigger strong emotional reactions in these children. Such a task might help to extract further information on how the symptoms associated with MCDD may or may not discriminate emotionprocessing ability from those with PDD-NOS.

\section{Strengths and Limitations}

To our knowledge this is the first study to directly compare children with MCDD to those with PDD-NOS (selected from a range of child psychiatric disorders) on socialcognitive functioning using well-validated research criteria for MCDD and PDD-NOS. A strength of this study was the selection of children with MCDD and PDD-NOS on the basis of explicit research criteria. Earlier studies on the neuro-cognitive profiles of children resembling MCDD included ill-defined groups representative of broad categories of disorder. Other studies examining symptom profiles and thought disorder using clearly defined groups of MCDD children selected these children from a sample of children with PDD, implying that MCDD can only occur within the broader category of PDD (van der Gaag et al. $1995,2005)$. We screened children from a larger sample of outpatients, and applied the research criteria for both MCDD and PDD-NOS independently from one another. Therefore, a diagnosis of MCDD could occur if the child did not meet criteria for PDD, consistent with earlier work suggesting that only approximately half of the children with MCDD also met criteria for PDD-NOS (Towbin et al. 1993). Furthermore, PDD-NOS has in many studies been assessed as a 'default' diagnosis of the DSM when children did not quite meet the diagnosis for any of the other PDD subtypes rather than being explicitly defined on its own (see Walker et al. 2004). Explicit research criteria and not a DSM default option were applied in this study to identify children with PDD-NOS.

However, we were also faced with a number of limitations. Since MCDD is not currently a DSM-IV diagnosis, all MCDD children had been assigned other clinical diagnoses. These diagnoses, based on DSM-IV and the DISC, included PDD-NOS, anxiety disorders (including separation anxiety and obsessive compulsive disorder), disruptive behavior disorders, in addition to ratings of psychotic thought problems (rated by the Child and Adolescent Functional Assessment Scale (CAFAS) and the CBCL thought problems subscale) (see also de Bruin et al. 2007). Approximately $40 \%$ of the MCDD children also met research criteria for PDD-NOS. Thus, although the PDDNOS children in this study did not meet criteria for MCDD, some of the children in the MCDD group also met the criteria for PDD-NOS. Our results of an advantage for the speed of recognizing neutral faces compared to complex patterns among children in the 'pure MCDD' group suggest that the more attention-demanding strategy of face recognition used by children with PDD-NOS may be dependent to a larger extent on their 'autistic-like qualities' rather than specific characteristics of MCDD. It is also noteworthy that these results emerged despite the small number of participants in this 'pure MCDD' group. Future studies could aim for a purer comparison by using groups of PDDNOS (without MCDD) and MCDD (without PDD-NOS). A further limitation was the relatively small sample size for the MCDD group. This was mainly due to the low prevalence rate of MCDD. However, another study assessing formal thought disorder in children with MCDD included a similar sample size (van der Gaag et al. 2005) and found higher rates of formal thought disorder in children with MCDD compared to clinical or healthy control groups. Furthermore, de Bruin et al. (2007) directly compared children with MCDD to PDD-NOS, and reported differences in symptom profiles using similar-sized groups while standard deviations in the dependent measures relative to their means (i.e. the variance coefficients) were greater in that study than those of, for example, the reaction times in our study. This suggests that the hypothesized group differences could have been smaller in order to be detected as significant. We are therefore not inclined to interpret the few significant differences in our study as being due to a power problem. We cannot exclude the possibility that 
children with MCDD and PDD-NOS use different cognitive strategies to achieve the same end level of performance. The use of neuro-imaging technologies and ERP studies would help to rule out this possibility, and may yield greater insight into the mechanisms underlying social cognition in these children.

\section{Conclusion}

Despite the existence of thought disorder in children with MCDD, the symptom level differences between PDD-NOS and MCDD children, and the biological/psychophysiological differences between MCDD children and other comparison groups, we found little evidence that children with MCDD are clearly distinguishable from those with PDD-NOS on the identification of facial expressions. Surprisingly, the high rates of anxieties and fears clinically characteristic of children with MCDD did not translate to any significant effects on our emotion-processing task. Further work is needed to probe whether more subtle emotion-processing differences exist. Such studies should focus on examining children's processing of emotional stimuli within a context more relevant to 'real-life' as well as detailed evaluation of children's emotion regulation ability.

The only significant difference to emerge was that children with MCDD not meeting the criteria for a PDD diagnosis demonstrated fewer errors and a faster processing of unfamiliar neutral faces compared to children with PDD-NOS who processed faces more similarly to how they processed complex patterns. This suggests a disadvantage in face processing being related to the autistic characteristics of the PDD-NOS. Based on these findings, it is recommended that the impact of autistic features (amount and severity) are carefully considered when evaluating a child with MCDD symptoms since such features may yield relevant information about the child's social cognitive abilities. Ideally, future work should include prospective designs which follow up children with MCDD who do and do not demonstrate autistic characteristics.

Acknowledgments This study was supported financially by a grant from the Netherlands Organization for Scientific Research (NWO/ ZonMw/OOG-100-002-006). We thank those children and families who participated in the study.

\section{References}

Ad-Dab'bagh, Y., \& Greenfield, B. (2001). Multiple complex developmental disorder: The "multiple and complex" evolution of the "childhood borderline syndrome" construct. Journal of the American Academy of Child and Adolescent Psychiatry, 40(8), 954-964.
APA. (1980). Diagnostic and statistical manual of mental disorders (3 ed.). Washington, DC: American Psychiatric Press.

APA. (1994). Diagnostic and statistical manual of mental disorders (4 ed.). Washington, DC: American Psychiatric Press.

Blair, R. J. (2003). Facial expressions, their communicatory functions and neuro-cognitive substrates. Philosophical Transactions of the Royal Society of London Series B, Biological Sciences, 358(1431), 561-572.

Buitelaar, J. K., \& van der Gaag, R. J. (1998). Diagnostic rules for children with PDD-NOS and multiple complex developmental disorder. Journal of Child Psychology and Psychiatry, 39(6), 911-919.

Buitelaar, J. K., van der Gaag, R., Klin, A., \& Volkmar, F. (1999a). Exploring the boundaries of pervasive developmental disorder not otherwise specified: Analyses of data from the DSM-IV Autistic Disorder Field Trial. Journal of Autism and Developmental Disorders, 29(1), 33-43.

Buitelaar, J. K., van der Wees, M., Swaab-Barneveld, H., \& van der Gaag, R. J. (1999b). Verbal memory and Performance IQ predict theory of mind and emotion recognition ability in children with autistic spectrum disorders and in psychiatric control children. Journal of Child Psychology and Psychiatry, 40(6), 869-881.

Castelli, F. (2005). Understanding emotions from standardized facial expressions in autism and normal development. Autism, 9(4), $428-449$.

Celani, G., Battacchi, M. W., \& Arcidiacono, L. (1999). The understanding of the emotional meaning of facial expressions in people with autism. Journal of Autism and Developmental Disorders, 29(1), 57-66.

Cohen, D. J., Volkmar, F. R., \& Paul, R. (1986). Issues in the classification of pervasive developmental disorders: History and current status of nosology. Journal of the American Academy of Child Psychiatry, 25(2), 158-161.

de Bruin, E. I., de Nijs, P. F., Verheij, F., Hartman, C. A., \& Ferdinand, R. F. (2007). Multiple complex developmental disorder delineated from PDD-NOS. Journal of Autism and Developmental Disorders, 37(6), 1181-1191.

de Sonneville, L. M. J. (1999). Amsterdam neuropsychological tasks: A computer aided assessment program. In: B. P. L. M. den Brinker, P. J. Beek, A. N. Brand, S. J. Maarse, \& L. J. M. Mulder (Eds.), Cognitive ergonomics, clinical assessment and computerassisted learning: Computers in Psychology (Vol. 6, pp. 204217). Lisse: Swets \& Zeitlinger.

de Sonneville, L. M., Verschoor, C. A., Njiokiktjien, C., Op het Veld, V., Toorenaar, N., \& Vranken, M. (2002). Facial identity and facial emotions: Speed, accuracy, and processing strategies in children and adults. Journal of Clinical and Experimental Neuropsychology 24(2), 200-213.

Hadwin, J. A., Donnelly, N., French, C. C., Richards, A., Watts, A., \& Daley, D. (2003). The influence of children's self-report trait anxiety and depression on visual search for emotional faces. Journal of Child Psychology and Psychiatry, 44(3), 432-444.

Happe, F. G. E. (1995). The role of age and verbal-ability in the theory of mind task-performance of subjects with autism. Child Development, 66(3), 843-855.

Herba, C., \& Phillips, M. (2004). Annotation: Development of facial expression recognition from childhood to adolescence: Behavioural and neurological perspectives. Journal of Child Psychology and Psychiatry, 45(7), 1185-1198.

Hobson, R. P., Ouston, J., \& Lee, A. (1988). Emotion recognition in autism: Coordinating faces and voices. Psychological Medicine, 18(4), 911-923.

Howell, D. C. (1997). Statistical methods for psychology (4th ed.). London: Duxbury Press.

Jansen, L. M., Gispen-de Wied, C. C., Gademan, P. J., De Jonge, R. C., van der Linden, J. A., \& Kahn, R. S. (1998). Blunted cortisol 
response to a psychosocial stressor in schizophrenia. Schizophrenia Research, 33(1-2), 87-94.

Jansen, L. M., Gispen-de Wied, C. C., Van der Gaag, R. J., ten Hove, F., Willemsen-Swinkels, S. W., Harteveld, E., et al. (2000a). Unresponsiveness to psychosocial stress in a subgroup of autistic-like children, multiple complex developmental disorder. Psychoneuroendocrinology, 25(8), 753-764.

Jansen, L. M., Gispen-de Wied, C. C., \& Kahn, R. S. (2000b). Selective impairments in the stress response in schizophrenic patients. Psychopharmacology (Berl), 149(3), 319-325.

Jansen, L. M., Gispen-de Wied, C. C., van der Gaag, R. J., \& van Engeland, H. (2003). Differentiation between autism and multiple complex developmental disorder in response to psychosocial stress. Neuropsychopharmacology, 28(3), 582-590.

Kemner, C., van der Gaag, R. J., Verbaten, M., \& van Engeland, H. (1999). ERP differences among subtypes of pervasive developmental disorders. Biological Psychiatry, 46(6), 781-789.

Klin, A., Sparrow, S. S., de Bildt, A., Cicchetti, D. V., Cohen, D. J., \& Volkmar, F. R. (1999). A normed study of face recognition in autism and related disorders. Journal of Autism and Developmental Disorders, 29(6), 499-508.

Lofgren, D. P., Bemporad, J., King, J., Lindem, K., \& O’Driscoll, G. (1991). A prospective follow-up-study of so-called borderline children. American Journal of Psychiatry, 148(11), 1541-1547.

Lord, C., Rutter, M., DiLavore, P. C., \& Risi, S. (1999). Autism diagnostic observation schedule-WPS (ADOS-WPS). Los Angeles, CA: Western Psychological Services.

Mogg, K., Philippot, P., \& Bradley, B. P. (2004). Selective attention to angry faces in clinical social phobia. Journal of Abnormal Psychology, 113(1), 160-165.

Phillips, M. L., Drevets, W. C., Rauch, S. L., \& Lane, R. (2003a). Neurobiology of emotion perception II: Implications for major psychiatric disorders. Biological Psychiatry, 54(5), 515-528.

Phillips, M. L., Drevets, W. C., Rauch, S. L., \& Lane, R. (2003b). Neurobiology of emotion perception I: The neural basis of normal emotion perception. Biological Psychiatry, 54(5), 504514.
Serra, M., Althaus, M., de Sonneville, L. M., Stant, A. D., Jackson, A. E., \& Minderaa, R. B. (2003). Face recognition in children with a pervasive developmental disorder not otherwise specified. Journal of Autism and Developmental Disorders, 33(3), 303-317.

Serra, M., Jackson, A. E., van Geert, P. L., \& Minderaa, R. B. (1998). Brief report: Interpretation of facial expressions, postures and gestures in children with a pervasive developmental disorder not otherwise specified. Journal of Autism and Developmental Disorders, 28(3), 257-263.

Stevens, J. (1992). Applied multivariate statistics for the social sciences (2nd ed.). Hillside, NJ: Erlbaum.

Towbin, K. E., Dykens, E. M., Pearson, G. S., \& Cohen, D. J. (1993). Conceptualizing "borderline syndrome of childhood" and "childhood schizophrenia" as a developmental disorder. Journal of the American Academy of Child and Adolescent Psychiatry, 32(4), 775-782.

van der Gaag, R. J., Buitelaar, J., van den Ban, E., Bezemer, M., Njio, L., \& van Engeland, H. (1995). A controlled multivariate chart review of multiple complex developmental disorder. Journal of the American Academy of Child and Adolescent Psychiatry, 34(8), 1096-1106.

van der Gaag, R. J., Caplan, R., van Engeland, H., Loman, F., \& Buitelaar, J. K. (2005). A controlled study of formal thought disorder in children with autism and multiple complex developmental disorders. Journal of Child and Adolescent Psychopharmacology, 15(3), 465-476.

van Engeland, H., \& van der Gaag, R. J. (1994). MCDD in childhood-a precursor of schizophrenic spectrum disorders. Schizophrenia Research, 11(2), 197-197.

Walker, E. (1981). Emotion recognition in disturbed and normal children: A research note. Journal of Child Psychology and Psychiatry, 22(3), 263-268.

Walker, D. R., Thompson, A., Zwaigenbaum, L., Goldberg, J., Bryson, S. E., Mahoney, W. J., et al. (2004). Specifying PDDNOS: A comparison of PDD-NOS, Asperger syndrome, and autism. Journal of the American Academy of Child and Adolescent Psychiatry, 43(2), 172-180. 